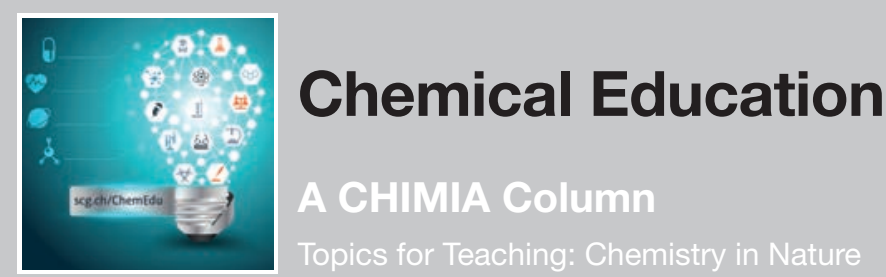

\section{Plant Toxins: Poison or Therapeutic?}

\section{Catherine E. Housecroft ${ }^{\star}$}

*Correspondence: Prof. C. E. Housecroft, E-mail: catherine.housecroft@unibas.ch, Department of Chemistry, University of Basel, BPR 1096, Mattenstrasse 24a, $\mathrm{CH}-4058$ Basel,

Abstract: Many plants that are classed as poisonous also have therapeutic uses, and this is illustrated using members of the Drimia and Digitalis genera which are sources of cardiac glycosides.

Keywords: Cardiac glycoside · Education · Plants Stereochemistry $\cdot$ Toxins

Earlier in this series of Chemical Education Columns, we introduced glycosides when describing anthocyanins. ${ }^{[1]}$ In the present article, we focus on cardiac glycosides which are compounds that stimulate the heart. Scheme 1 shows the basic building blocks of the two main classes of cardiac glycosides: bufadienolides and cardenolides. The prefix card- stems from the Greek for heart ( $\alpha \rho \delta \delta \alpha$, kardiá), while buf- originates from the fact that toads (genus Bufo) are one of the major sources of these compounds. ${ }^{[2]}$ The ending -enolide refers to the lactone unit (a cyclic carboxylic ester, top right in each structure in Scheme 1). The four-ring unit common to bufadienolides and cardenolides is characteristic of a steroid, although some steroids such as testosterone contain an unsaturated 6-membered ring. Cardiac glycosides inhibit the activity of the enzyme $\mathrm{Na}^{+} / \mathrm{K}^{+}$-ATPase, causing an increase in the intercellular $\mathrm{Na}^{+}$ion concentration which leads to stronger muscle contractions of the heart.

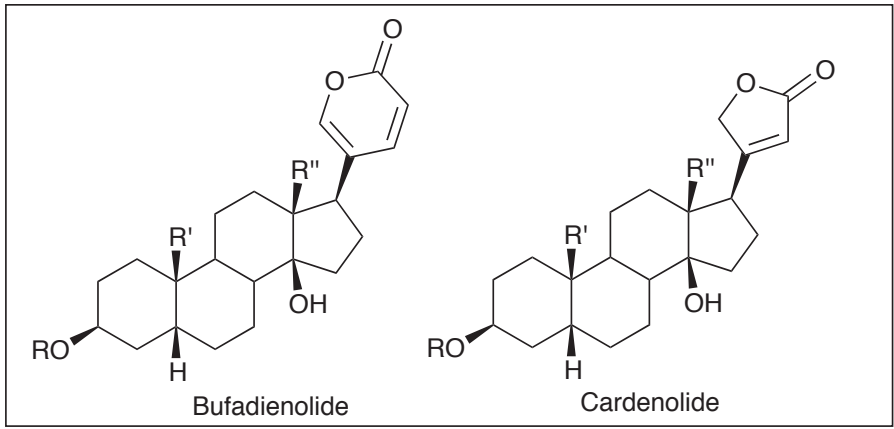

Scheme 1. The fundamental structures of bufadienolides and cardenolides.

A large range of bufadienolide and cardenolide compounds can be formed by variation in the $\mathrm{R}, \mathrm{R}^{\prime}$ and $\mathrm{R}^{\prime \prime}$ groups in the structures in Scheme 1 as well as by the introduction of glycosyl[1] units. Scheme 2 shows two examples. A remarkably large number of plants contain cardiac glycosides including members of the Digitalis genus (foxgloves, Fig. 1) and Drimia or Urginea genus (poison squills). An example of a poison squill is Drimia
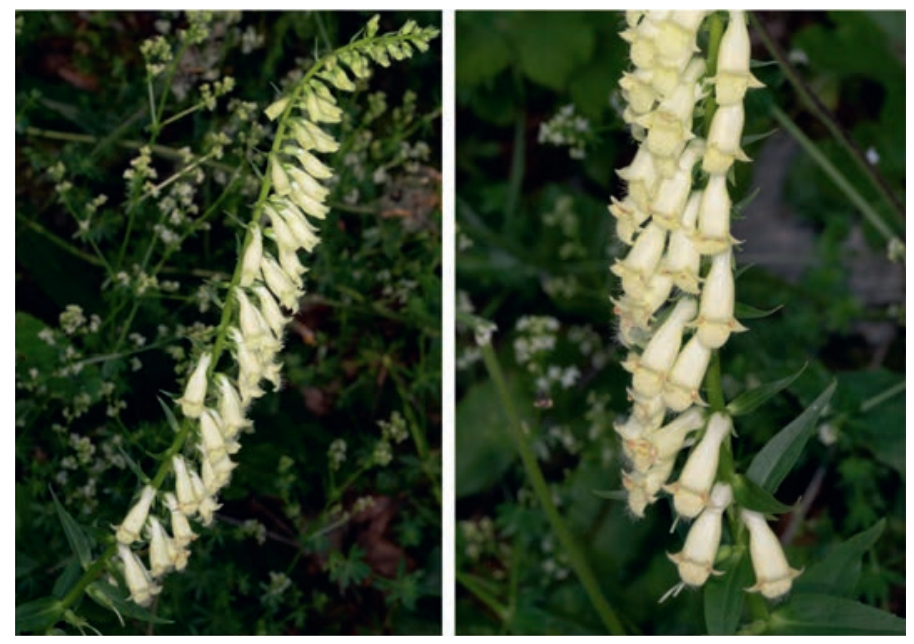

Fig. 1. Digitalis lutea (small yellow foxglove). CEdwin C. Constable 2019

altissima (tall white squill, Fig. 2) which is widely distributed in the savanna and open scrubland of sub-Saharan Africa. D. maritima (sea, maritime or red squill) grows in coastal regions of the Mediterranean, and its medicinal properties were described as early as $1500 \mathrm{BCE}$. In the Indian subcontinent, the expectorant, cardiostimulant, diuretic, emetic and nauseating effects of D. indica were described in the Bhavprakash Nighantu (Indian Materia Medica of Shri Bhavamishra) written in the $16^{\text {th }}$ century. ${ }^{[3]}$ Poison squills have large, onion-like bulbs which usually protrude above the ground (Fig. 2, right). Cardiac glucosides are found in all parts of poison squills, but the highest concentrations are in the bulbs.
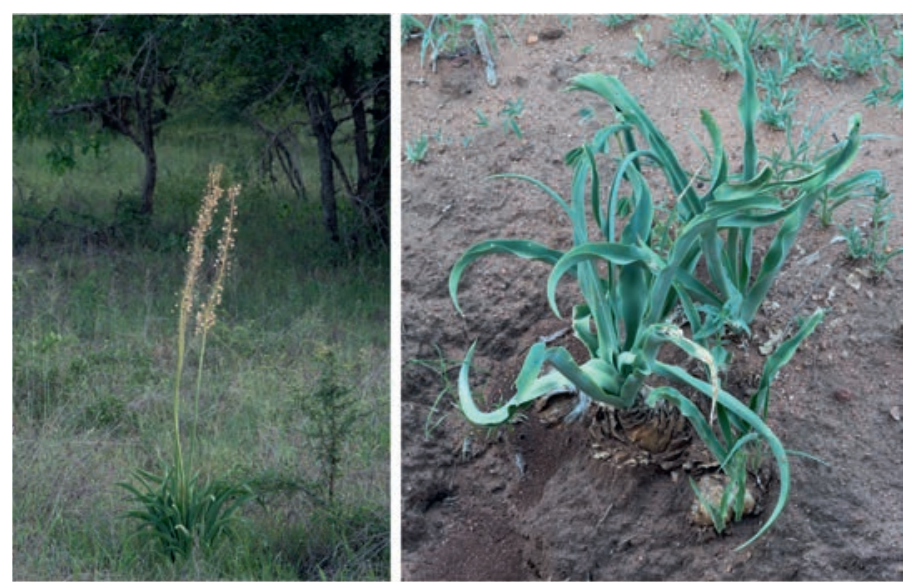

Fig. 2. Drimia altissima (tall white squill). CEdwin C. Constable 2020

Digoxin (Scheme 2) is a cardenolide glycoside and is isolated from Digitalis lanata (the woolly foxglove). It is an immensely important drug for the treatment of congestive heart failure. Once administered to a patient, it acts rapidly (within 30 minutes after 


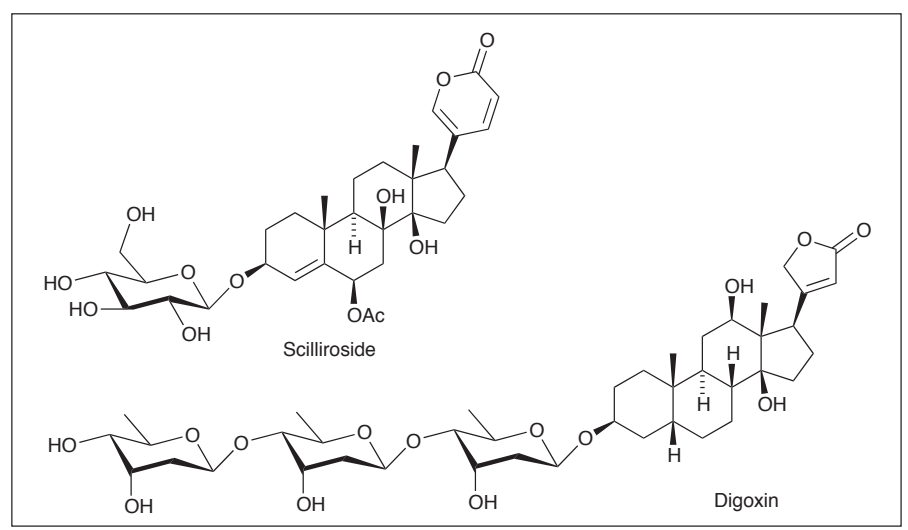

Scheme 2. The structures of scilliroside and digoxin, two examples of cardiac glycosides.

an intravenous injection). Scilliroside (Scheme 2) is a bufadienolide glucoside and is present in Drimia maritima. It used to be utilized as a rat poison, but is no longer manufactured for this purpose. The toxic effects of bufadienolide glucosides in species of Drimia have a significant impact on agriculture. In South Africa in the late 1990s, it was estimated that bufadienolide glucosides from plants accounted for the deaths of up to 12,000 head of cattle per year. ${ }^{[4]}$ Poisoning of domestic animals by bufadienolide glucoside-containing plants including $D$. altissima continues to have a substantial economic impact. ${ }^{[5]}$

The structures of cardiac glycosides provide an excellent opportunity to discuss stereochemistry, and in particular the stereochemistry of the steroid unit. Three of the four rings in the steroid building blocks in Scheme 1 are saturated, 6-membered rings. We start therefore, with the stereochemistry of cyclohexane $\left(\mathrm{C}_{6} \mathrm{H}_{12}\right)$. The structure of cyclohexane is shown at the top of Scheme 3 in a chair conformation. Partial rotation about the $\mathrm{C}-\mathrm{C}$ bonds in cyclohexane 'flips' the ring between the chair, twist-boat and boat conformations, and the lowest energy conformation is the chair: $E$ (chair) $<<E$ (twist-boat) $<E$ (boat). The diagrams at the top of Scheme 3 also highlight the axial and equatorial positions of the $\mathrm{H}$ atoms in cyclohexane. The Newman projection on the right views the structure along the two $\mathrm{C}-\mathrm{C}$ bonds shown in bold in the left-hand diagram. Now consider the structure of the bicyclic molecule decahydronaphthalene $\left(\mathrm{C}_{10} \mathrm{H}_{18}\right.$, bottom of Scheme 3 ). Fusion of two $\mathrm{C}_{6}$ rings, each in a chair conformation, can be through either two equatorial positions, or one axial and one equatorial position. This leads to either the trans- or cis-diastereoisomers of decahydronaphthalene, two representations of which are shown in Scheme 3. The stereochemistry of the $\mathrm{H}$ atoms at the point of ring fusion distinguishes the two diastereoisomers.

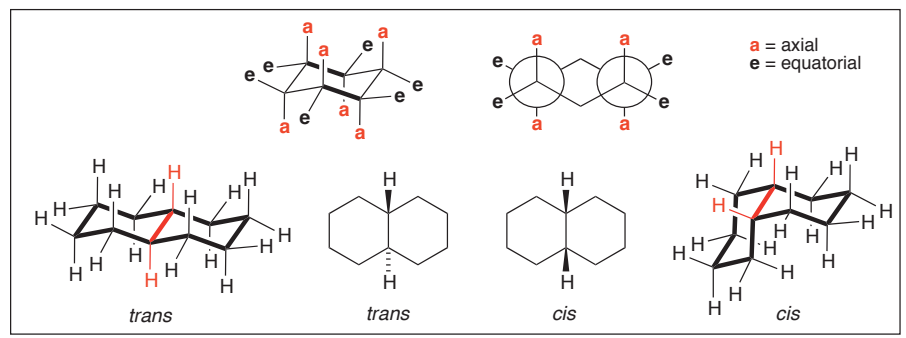

Scheme 3. Top: The chair conformation of a cyclohexane ring showing the axial and equatorial positions of the $\mathrm{H}$ atoms, and a Newman projection of the same structure viewed along the two $\mathrm{C}-\mathrm{C}$ bonds shown in bold in the left-hand diagram. Bottom: The trans- and cis-diastereoisomers of decahydronaphthalene $\left(\mathrm{C}_{10} \mathrm{H}_{18}\right)$ highlighting the stereochemistry of the $\mathrm{H}$ atoms at the position of ring fusion.

Now consider the steroid building block shown in Scheme 1; it contains three 6-membered rings and one 5-membered ring.
Scheme 4 shows the three possible conformers. The trans-transtrans diastereoisomer is found in most naturally occurring steroids, while a smaller number of natural steroids, including cholic acids, are the cis-trans-trans diastereoisomer. Only a few natural steroids possess cis-trans-cis ring fusion and these include the cardiac glycosides. Fig. 3 shows the crystallographically determined structure of digoxin. ${ }^{[6]}$ Compare this with the schematic representation in Scheme 2.

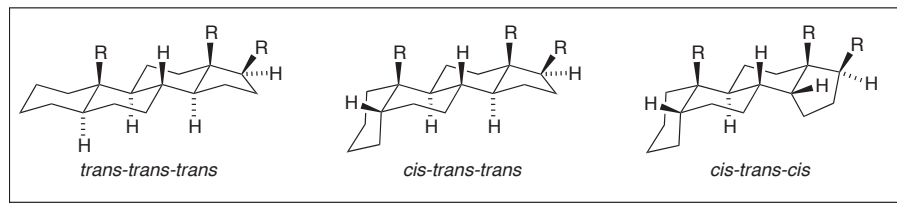

Scheme 4. The three possible ring fusions in a steroid.

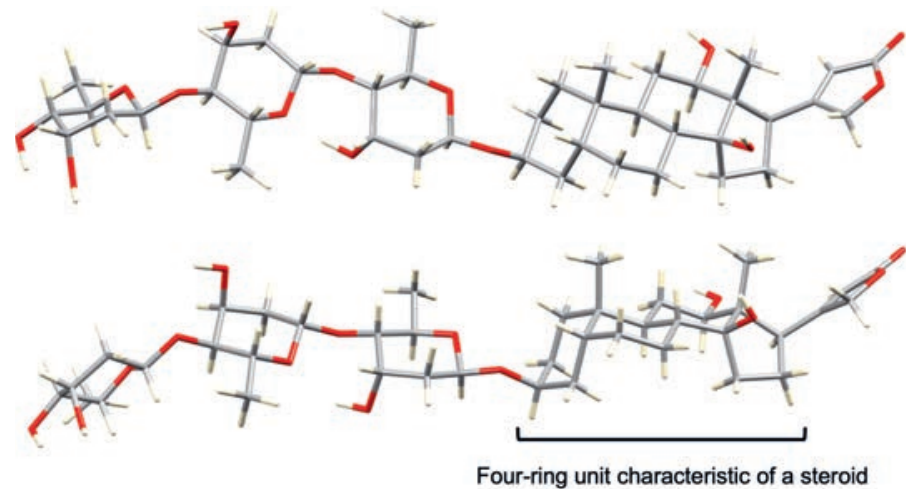

Fig. 3. Two views of a molecule of digoxin, showing the steroid building block and the chair-conformations of all the 6-membered rings. (Cambridge Structural Database refcode DIGOXN10).

Returning to the toxic effects of bufadienolide glucosides, it is relevant to consider the words of the Swiss physician and alchemist Paracelsus (1493-1541) who is regarded as the Father of Toxicology: "Solely the dose determines that a thing is not a poison". ${ }^{[7]}$ Whereas plants including Drimia altissima can be fatal to grazing cattle in southern Africa, poison squills in the Drimia genus have been used since ancient times as a source of drugs for medicinal applications. Bufadienolide glycosides extracted from D. maritima are one of the oldest known heart stimulants. ${ }^{[8]}$ Many plants that are considered to be poisonous have therapeutic uses, both in traditional and modern medicines.

This article highlights plants, illustrated using members of the Drimia and Digitalis genera, that are sources of cardiac glycosides. This also provided the opportunity to introduce the stereochemistry associated with the formation of fused ring systems, in particular in steroids.

Received: March 27, 2020

[1] C. E. Housecroft, Chimia 2019, 9, 760.

[2] H. Gao, R. Popescu, B. Kopp, Z. Wang, Nat. Prod. Rep. 2011, 28, 953.

[3] S. Aswal, A. Kumar, R.B. Semwal, A. Chauhan, A. Kumar, J. Lehmann, D.K. Sewal, Medicina 2019, 55, 255.

[4] P.S. Steyn, F. R. van Heerden, Nat. Prod. Rep. 1998, 15, 397.

[5] C. Botha, Molecules 2016, 21, 348.

[6] K. Go, G. Kartha, J. P. Chen, Acta Crystallogr. 1980, 36B, 1811.

[7] P. Grandjean, Basic Clin. Pharm. Toxicol. 2016, 119, 126.

[8] H. Bucht, J. Ek, H. Eliasch, B. Thomasson, L. Werkö, Am. Heart J. 1957, 54,376 .

This column is one of a series designed to attract teachers to topics that link chemistry to Nature and stimulate students by seeing real-life applications of the subject. 